\title{
INTERPOLATION IN BANACH SPACES
}

0. Introduction. Let $X$ be a compact Hausdorff space and $Y$ a closed subset of $X$. Let $A$ be a closed subspace of $C(X)$ (complex-valued continuous functions with uniform norm) and $M$ a subspace of $C(Y)$. A typical interpolation problem involves finding, for each $g \in M$, an $f \in A$ such that $\left.f\right|_{Y}=g$. More particularly one may require $\|f\|_{X} \leqq c\|g\|_{Y}(c \geqq 1)$.

The various formulations of conditions assuring solutions to interpolation problems have come to be called generalized Rudin-Carleson theorems and usually are expressed in terms of the regular complex Borel measures on $X$ (elements of $\left.C(X)^{*}\right)$ that annihilate $A$. Indeed, the results of Rudin [21] and Carleson [12] deal with measures on the unit circle $T \subset \mathrm{C}$ that annihilate the disk algebra (elements of $C(T)$ with continuous extensions analytic on the interior of the unit disk).

In this paper we bring together a variety of interpolation results and develop them as consequences of the geometric structure of polar sets in $A^{*}$. This approach is very much in the spirit of Ando [4] where the emphasis is on so-called split sets and their polar properties.

One feature of earlier studies by, for example Bishop [9], Gamelin [15], Glicksberg [16] and others is the connection between peak-sets (or generalized peak-sets) and norm-preserving interpolation. Here we show that both the peaking property and the interpolation property are consequences of a geometric structure in the dual, $A^{*}$, which we term $d e$ composability. This is a weak version of splittability, and does not require the presence of the projection in $A^{*}$ that is associated with split sets. The relation of decomposable cones and peak-sets has been noted earlier [6] and the emphasis here is on the decomposition of the dual ball and other bounded sets in $A^{*}$.

Our approach is to treat interpolation as a phenomenon involving a Banach space $E$ and its dual $E^{*}$. We consider a weak* compact convex set $\hat{Y}$ containing 0 in $E^{*}$ and identify elements $f \in E$ (via their restrictions under the standard duality of $E, E^{*}$ ) with a dense subspace of $A_{0}(\hat{Y})$, the continuous complex-homogeneous affine functions on $\hat{Y}$ (details in $\S 2$ ). If $V$ is a closed convex neighborhood of 0 in $A_{0}(\hat{Y}), A$ a closed convex cone, and $B$ a closed convex bounded subset containing 0 in $E$ then we define the interpolation problem involving $A, B$ and $V$ :

Received by the editors on October 31, 1977.

AMS Subject Classification: Primary 46A20, 46E15; Secondary $46 \mathrm{~J} 10$.

Key Words: Interpolation, split sets, Rudin-Carleson theorems.

Copyright (c) 1979 Rocky Mountain Mathematical Consortium 


$$
\begin{aligned}
& \text { given } g \in A \text { with }\left.g\right|_{\hat{Y}} \in V \subset A_{0}(\hat{Y}) \text { find } \\
& f \in B \subset E \text { such that }\left.f\right|_{\hat{Y}}=g
\end{aligned}
$$

Our basic machinery consists of the gauge lemma of [8] (we present a slightly modified version here), developed in $\S 1$. In $\S 2$ we formulate the above interpolation set-up and present criteria for what we term approximate solutions. This is closely related to the standard characterizations derived from the open mapping and closed range theorems (e.g. [18], [22]). For unity of treatment we deduce these from the gauge lemma. We next consider the problem of, given approximate solutions, when do exact solutions exist? Again, sufficient conditions are presented using the gauge lemma. In $\S 3$ we introduce the polar geometric properties (principally decomposability) that yield exact solutions. This is done in a fairly abstract setting. $\S 4$ then relates these conditions to some of the standard $C(X)$ theory and in particular the case where $A$ is an algebra of functions.

1. Preliminaries, Notation and the Gauge Lemma. Let $A$ be a subset of the normed linear space $(E,\|\cdot\|)$. We denote

$$
A_{r} \triangleq\{x \in A:\|x\| \leqq r\} \text {. }
$$

Thus the unit ball of $E$ is $E_{i}$, etc. We refer to bounded closed convex neighborhoods of 0 in $E$ as 0-neighborhoods.

If $A$ is a convex set containing 0 , we denote the Minkowski functional of $A$ by $p_{A}$ or $p(A)$. Thus

$$
p_{A}(x)=\inf \{r \geqq 0: x \in r A\} .
$$

If $p$ is a positively homogeneous, sub-additive functional such that

$$
\sum_{n=1}^{\infty} p\left(x_{n}\right)<\infty \text { implies } x=\sum_{n=1}^{\infty} \text { exists and } p(x) \leqq \sum_{n=1}^{\infty} p\left(x_{n}\right)
$$

then $p$ is called a gauge. It is the Minkowski functional of

$$
B=\{x \in E: p(x) \leqq 1\}
$$

where $B$ is a complete bounded convex set containing 0 . Conversely the Minkowski functional of such a set is a gauge.

If $p$ has the weaker property that

$$
p(x) \leqq \sum_{n=1}^{\infty} p\left(x_{n}\right) \text { whenever } x=\sum_{n=1}^{\infty} x_{n} \text { exists }
$$


then $p$ is called a pre-gauge.

For example, the Minkowski functional of a closed (but possibly unbounded) convex set containing 0 is a pre-gauge. Other examples are:

(a) the Minkowski functional of the sum of two closed convex sets containing 0 , one of which is complete and bounded;

(b) the Minkowski functional of the image of a complete and bounded convex set containing 0 under a bounded linear map.

In general we say a convex set $B$ containing 0 is a gauge set, or a pre-gauge set, if the Minkowski functional $p_{B}$ has the corresponding property.

To formulate the fundamental gauge lemma we need the notion of the distance from a point to a set via a gauge set. Thus we define the distance to $A$ via $B$ by

$$
d(A, B)(x) \triangleq \inf \{r: \mathrm{x} \in A+r B\} .
$$

We will abbreviate this by $d_{B}$, or just $d$, if the sets $A$ and $B$ are understood. We also require the closure of $d$, given by

$$
\bar{d}(A, B)=\inf \left\{r: x \in(A+r B)^{-}\right\} .
$$

Finally, we require a refinement of $\bar{d}$, namely the $C$-closure of $d(A, B)$, given by

$$
\bar{d}(A, B ; C)=\inf \{r: x \in A+r B+\epsilon C, \text { for all } \epsilon>0\} .
$$

If $C$ is a 0 -neighborhood then $\bar{d}(A, B ; C)=\bar{d}(A, B)$.

The gauge lemma gives various sufficient conditions for $d(A, B)=\bar{d}(A, B ; C)$ on a domain set $D$.

Gauge Lemma 1.1. Let $A, B, C, D$ be convex subsets of a normed linear space $E$, all containing 0 , and satisfying the following hypotheses:

(1) the domain $D$ contains the (not necessarily closed) subspace spanned by $B$,

(2) $C$ is closed and bounded,

(3) $\bar{d}(A, B ; C) \leqq \alpha d(A, C)$ on $D$ for some $\alpha>0$,

(4) any one of

(i) $A$ is closed and $P_{B}$ is a pre-gauge, or

(ii) $A$ is compact and $p_{B}$ is a re-gauge, or

(iii) $A$ is complete, $B$ is bounded and $p_{B}$ is a pre-gauge.

Then $d(A, B)=\bar{d}(A, B ; C)$ on $D$.

Proof. We write $d, \bar{d}$ for the distance from $A$ via $B$ and the $C$-closure of the distance, respectively, and $d_{C}$ for the distance to $A$ via $C$. We have $\bar{d} \leqq d$ by definition. Let $x_{0} \in D, \bar{d}\left(x_{0}\right) \leqq r_{0}<r<\infty$. Choose 
a sequence $\left(r_{n}\right)_{n=1}^{\infty}$ of positive numbers with

$$
r_{1}>r_{0} \text { and } \sum_{n=1}^{\infty} r_{n}<r
$$

Since $\bar{d}\left(x_{0}\right) \leqq r_{0}$ we have

$$
x_{0}=a_{1}+b_{1}+c_{1} ; a_{1} \in A, p_{B}\left(b_{1}\right)<r_{1} \text { and } p_{c}\left(c_{1}\right)<r_{2} / \alpha .
$$

Then, by (1), $x_{0}-b_{1} \in D$ and $x_{0}-b_{1}=a_{1}+c_{1}$. Hence $d_{c}\left(x_{0}-b_{1}\right)<r_{2} / \alpha$ so that, by (3), $\bar{d}\left(x_{0}-b_{1}\right)<r_{2}$. Hence

$$
x_{0}-b_{1}=a_{2}+b_{2}+c_{2} ; a_{2} \in A, p_{B}\left(b_{2}\right)<r_{2} \text { and } p_{C}\left(c_{2}\right)<r_{3} / \alpha .
$$

Continuing by induction, we obtain sequences $\left(a_{n}\right),\left(b_{n}\right)$ and $\left(c_{n}\right)$ such that

$$
\begin{gathered}
a_{n} \in A, p_{B}\left(b_{n}\right)<r_{n}, p_{C}\left(c_{n}\right)<r_{n+1} / \alpha \text { and } \\
x_{0}=a_{n}+\left(b_{1}+\cdots+b_{n}\right)+c_{n} .
\end{gathered}
$$

By (2) we have $\left\|c_{n}\right\| \rightarrow 0$. If (i) holds then there exists

$$
b=\sum_{n=1}^{\infty} b_{n} \text { with } p_{B}(b) \leqq \sum_{n=1}^{\infty} p_{B}\left(b_{n}\right)<r
$$

and hence $a_{n} \rightarrow a \in A$. Thus $x_{0} \in A+r B$. If (ii) holds then a subsequence $a_{m} \rightarrow a \in A$ and therefore $\sum_{n=1}^{\infty} b_{n}=b$ exists and $p_{B}(b)<r$.

In case of (iii) we have $a_{n+1}-a_{n}=b_{n+1}+c_{n+1}-c_{n}$ so that $\left\|a_{n+1}-a_{n}\right\| \leqq \beta r_{n+1}+\gamma / \alpha\left(r_{n+1}+r_{n}\right)$, where $\beta, \gamma$ are bounds on $B$ and $C$. Thus $\left(a_{n}\right)$ is Cauchy so that $a_{n} \rightarrow a \in A$ and consequently $b=\sum_{n=1}^{\infty} b_{n}$ exists with $p_{B}(b)<r$.

In making applications of the gauge lemma we shall adopt the convention of writing $A \longleftrightarrow{ }^{\circ}, B \longleftrightarrow{ }^{\circ}$, etc. where we fill in the blanks with the names of the sets currently playing the roles of $A, B, C$, and $D$ in the standard form in the gauge lemma given above. The meaning of $d$ and $\bar{d}$ will follow accordingly.

For the first application we are concerned with conditions under which the Minkowski functionals of a set $B$ and its closure $\bar{B}$ coincide. We say the $D$-interior of $B$, denoted $D$-int $(B)$, is nonempty if for some $\alpha>0$

$$
D \cap U \subset \alpha B
$$

where $U$ is the unit ball of $E$. 
THEOREM 1.2. Let $D$ be a closed convex cone and let $B$ be a pregauge set with $\pm B \subset D$. If $D$-int $(\bar{B}) \neq \varnothing$ then $D$-int $(B) \neq \phi$ and

$$
p_{B}=p(\bar{B}) \text { on } D \text {. }
$$

Proof. We apply the gauge lemma with

$$
\begin{aligned}
& A \leftrightarrow\{0\} \\
& B \longleftrightarrow B \\
& C \longleftrightarrow U \\
& D \longleftrightarrow D .
\end{aligned}
$$

Then $d, \bar{d}$ and $d_{C}$ are the functionals $p_{B}, p(\bar{B})$ and $\|\circ\|$ so that

$$
D \cap U \subset \alpha \bar{B}
$$

implies $\bar{d} \leqq \alpha d_{C}$ on $D$. Thus, using (4ii), the conclusion follows.

It is convenient to say $B$ is D-regular if $p_{B}=p(\bar{B})$ on $D$. This just amounts to the condition

$$
D \cap \bar{B}=\bigcap_{\lambda>1}[D \cap(\lambda B)] .
$$

Corollary 1.3. Let $A$ and $D$ be closed convex cones and let $B$ be a complete and bounded convex set containing 0 . If $\pm A \subset D$ and $+B \subset D$ and $D$-int $(\overline{A+B}) \neq \varnothing$ then $A+B$ and $A_{r}+s B$ are D-regular sets for all $r, s>0$.

Proof. Since $D \cap U \subset \alpha(\overline{A+B})=\overline{A+\alpha B}$ for some $\overline{s \bar{B}}) \neq \phi$ for any $r, s>0$. Since $B$ is complete and bounded, $A+B$ and $A_{r}+s B$ are pre-gauge sets satisfying the hypothesis of Theorem 1.2.

For $A \subset E$ we define the polar, $A^{\circ} \subset E^{*}$ by

$$
A^{\circ}=\left\{x^{*} \in E^{*}: \operatorname{re}\left(x, x^{*}\right) \leqq 1\right\} .
$$

If $B \subset E^{*}$ then, unless otherwise specified, $B^{\circ}$ will always refer to the subset of $E$ defined dually.

We shall assume the various results of the polar calculus, noting in particular that for $A$ closed and convex containing 0 that

$$
p_{A}(x)=\sup \left\{\operatorname{re}\left(x, x^{*}\right): x^{*} \in A^{\circ}\right\} .
$$

The right hand side defines the support function $\rho_{A^{\circ}}$ at the point $x \in E$. Dually

$$
\rho_{A}=p_{A^{\circ}} .
$$


Also, if $\theta: \mathrm{E} \rightarrow F$ is a bounded linear map whose adjoint $\theta^{*}: F^{*} \rightarrow E^{*}$ (given by $\left.\left(\theta x, y^{*}\right)=\left(x, \theta^{*} y^{*}\right)\right)$ then

$$
(\theta A)^{\circ}=\left(\theta^{*}\right)^{-1}\left(A^{\circ}\right) \text { and }\left[\theta^{*}(B)\right]^{\circ}=\theta^{-1}\left(B^{\circ}\right) .
$$

If $0 \in A$, a closed convex set, we denote

$$
A^{\prime}=\left\{x: p_{A}(x)<1\right\} .
$$

2. The Interpolation Problem. We turn now to the interpolation problem of the introduction. First take $\hat{Y}$ to be a weak* compact convex balanced set in $E^{*}$ and let the restriction map $\theta$ be given by

$$
\theta: E \rightarrow A_{0}(\hat{Y}) ; \quad \theta f(y)=(f, y) .
$$

Define

$$
\Phi: \hat{Y} \rightarrow A_{0}(\hat{Y})^{*} ; \quad \Phi(y)(g)=g(y) .
$$

Let $N=\cup_{n=1}^{\infty} n \hat{Y}$. Since $\hat{Y}$ is convex and balanced, $N$ is a (complex) subspace of $E^{*}$. The standard identification of $N$ and $A_{0}(\hat{Y})^{*}$ is summarized in the following.

THEOREM 2.1.

(1) $\Phi$ is a complex homogeneous affine homeomorphism of $\hat{Y}$ with the unit ball $A_{0}(\hat{Y})_{1}{ }^{*}$ (weak* topologies),

(2) $\Phi$ extends to an isomorphism (also denoted $\Phi)$ of $N$ onto $A_{0}(\hat{Y})^{*}$,

(3) $\theta$ is a bounded one-to-one map with $\Phi^{-1}=\theta^{*}$,

(4) the range $\theta E$ is dense in $A_{0}(\hat{Y})$.

Proof. The fact that $\Phi$ is affine and homogeneous, hence extending to $N$, is immediate from its definition. Since

$$
(\theta f, \Phi y)=(f, y) ; f \in E, y \in \hat{Y},
$$

$\Phi$ is one-to-one.

If $g \in A_{0}(\hat{Y})$ then

$$
\|g\|=\sup \{|g(y)|: y \in \hat{Y}\}=\sup \{\operatorname{re}(g, \Phi y): y \in \hat{Y}\}
$$

and hence the separation theorem yields $\Phi(\hat{Y})=A_{0}(\hat{Y})_{1}{ }^{*}$. This completes (1) and (2); (3) is immediate from the duality relations $\left(E, E^{*}\right)$ and $A_{0}(\hat{Y}), A_{0}(\hat{Y})^{*}$. For $(4)$,

$$
(\theta E)^{\circ}=\left(\theta^{*}\right)^{-1}(0)=\{\Phi(0)\}=\{0\} .
$$

In view of Theorem $2.1(4)$ it is clearly pertinent to determine when the map $\theta$ takes $E$ onto $A_{0}(\hat{Y})$ (has closed range in $C(\hat{Y})$ ). The open mapping theorem says that $\theta$ is onto if and only if $\theta$ takes bounded 
neighborhoods of 0 to neighborhoods of 0 . This is incorporated in the following list of equivalences.

Theorem 2.2. Let $\theta: E \rightarrow F$ be a bounded linear map between $B a-$ nach spaces with $\theta E$ dense in $F$. The following are equivalent:

(i) $\theta$ is onto (has closed range),

(ii) $F_{1} \subset \overline{\theta\left(E_{r}\right)}$ (some $r>0$ ),

(iii) $F_{1} \subset \theta\left(E_{s}\right)($ all $s>r)$,

(iv) $(\theta E) \cap F_{1} \subset \theta\left(E_{s}\right)(s>r)$,

(v) $\theta^{*}$ has $w^{*}$ closed range $N$ (spanned by $\left.\hat{Y}=\theta^{*}\left(F_{1}{ }^{*}\right)\right)$,

(vi) $\theta^{*}$ has norm closed range $N$ (spanned by $E_{1}{ }^{*} \cap N$ ),

(vii) $N \cap E_{1}^{*} \subset r \hat{Y}$ (some $r>0$ ).

Proof. (ii) follows from (i) by the standard Baire Category argument. Then (ii) implies (iii) is the $1 / 2^{n}$ argument of the Open Mapping Theorem. Alternatively, the gauge lemma can be applied with

$$
D \longleftrightarrow F, A \longleftrightarrow\{0\}, \mathrm{B} \longleftrightarrow \theta\left(E_{1}\right) \text { (a pre-gauge), } C \longleftrightarrow F_{1} \text {. }
$$

Then (ii) says $\bar{d} \leqq \alpha d_{C}$ and (iii) follows from $d=\bar{d}$. (iii) implies (iv) is trivial and (iv) implies, for $\alpha<\beta<1$,

$$
[\overline{\theta(E)}]_{\alpha} \subset \overline{(\theta E)}{ }_{\beta} \subset \overline{\theta E_{r}} .
$$

Thus $[\overline{\theta E}]_{1} \subset \theta E_{s}$ (by (ii) implies (iii)) so that

and (i) follows.

$$
\overline{\theta E}=\theta E
$$

That (v) implies (vi) is clear and (vi) implies (vii) follows from (i) implies (iv) applied to the dual spaces. If (vii) holds then

$$
N \cap E_{1}^{*}=(r \hat{Y}) \cap E_{1}^{*}
$$

is weak* compact so that $N$ is weak* closed by the Krein-Smulyan Theorem. Finally (vii) is equivalent to

$$
\left(\theta^{*}\right)^{-1}\left(E_{1}{ }^{*}\right) \subset r F_{1}^{*}
$$

which, by the polar calculus, is equivalent to (ii).

Corollary 2.3. If $\theta: E \rightarrow F$ has closed range and $A \subset \theta E$ then

$$
\overline{\theta^{-1}(\bar{A})}=\theta^{-1}(\bar{A}) \text {. }
$$

Proof. Let $Z=\theta E$ and note that

$$
\bar{A} \cap Z=\overline{A \cap Z}=\bigcap_{\epsilon>0}\left(A \cap Z+\theta E_{\epsilon}\right)
$$

by (iii) above. 
In general if $Y$ is weak* compact in $E^{*}$ and $\hat{Y}=\operatorname{cl}$-conv bal $(Y)$ then, by Theorem 2.2,E $\left.\right|_{Y}$ is closed in $C(Y)$ if and only if $N=\cup_{n=1}^{\infty} n \hat{Y}$ is weak* closed in $E^{*}$. In this case we say $Y$ is an interpolation set for $E$.

Given a bounded closed convex neighborhood $V$ of 0 in $A_{0}(\hat{Y})$ the interpolation problem involving sets $A, B \subset E$ and $V \subset A_{0}(\hat{Y})$ is:

(*) $\quad$ given $g \in A$ with $\left.g\right|_{\hat{Y}} \in V$ find $f \in B$ such that $\left.f\right|_{\hat{Y}}=\left.g\right|_{\hat{Y}^{*}}$

In the sequel we shall speak of $\operatorname{IP}(*)$ for $\hat{Y}$ involving $A, B$ and $V$.

Let $p_{B}$ and $p_{V}$ be the Minkowski functionals of $B \subset E$ and $V \subset A_{0}(\hat{Y})$. We say the interpolation problem has approximate solutions if $g \in A$ and $p_{V}(g \mid \hat{Y})<r$ implies there is an $f$ such that

$$
p_{B}(f)<r \text { and }\left.f\right|_{\hat{Y}}=\left.g\right|_{\hat{Y}}
$$

We say exact solutions exist if $g \in A$ and $p_{V}(g \mid \hat{y}) \leqq r$ implies there is an $f$ such that

$$
p_{B}(f) \leqq r \text { and }\left.f\right|_{\hat{y}}=\left.g\right|_{\hat{Y}}
$$

Corollary 2.4. Let $Y$ be weak* compact in $E^{*}$ and $\hat{Y}=w^{*} \mathrm{cl}-$ $\operatorname{conv} \operatorname{bal}(Y)$. The following are equivalent:

(i) $N=\cup_{n=1}^{\infty} n \hat{Y}$ is $w^{*}$-closed,

(ii) $Y$ is an interpolation set,

(iii) $\left.E\right|_{Y}$ is isomorphic to $A_{0}(\hat{Y})$ and for each closed convex bounded neighborhood $V$ of 0 in $A_{0}(\hat{Y})$ there is a bounded convex neighborhood $U$ of 0 in $E$ such that the interpolation problem involving $E, U$ and $V$ has solutions.

We note that $N$ is weak* closed (equivalently, $Y$ is an interpolation set) if and only if $N=K^{\circ}$, where

$$
K=\operatorname{ker} \theta=\left\{f \in E:\left.f\right|_{Y} \equiv 0\right\} .
$$

The archetype upon which the abstract setting is modeled involves $C(X), X$ compact Hausdorff, and $Y$ a closed subset of $X$. Now $\theta$ is the restriction map from $C(X)$ to $C(Y)$ and $K=\operatorname{ker} \theta$. If $U$ is the unit ball (uniform norm) of $C(X)$ then (denoting $\theta U$ by $\left.U\right|_{Y}$ ), $\left.U\right|_{Y}$ is the unit ball of $C(Y)$ (by Urysohn's lemma).

Also $K^{\circ}$ is isometrically equivalent to $C(Y)^{*}$ (the regular Borel measures on $X$ with support in $Y$ ) and $K^{\circ}$ is the (algebraic) span of $\hat{Y}=w^{*}$ cl-conv bal $(\phi Y) ; \phi: X \rightarrow C(X)^{*}$ the evaluation map.

Now let $A$ be a closed cone in $C(X)$ and consider the problem of finding, for $g \in A$ and $\left\|\left.g\right|_{Y}\right\| \leqq 1, f \in A$ with $f_{Y}=\left.g\right|_{Y}$ and $\|f\| \leqq 1$. If we take $\hat{Y}=U^{\circ} \cap K^{\circ}$ then this translates directly to the interpolation problem involving $A, U \cap A$ and $V$, where $V$ is the unit ball of $A_{0}(\hat{Y})$. 
Since $\theta^{-1} V=K+U$, this problem has exact solutions if and only if

$$
A \cap(K+U) \subset A \cap U+K .
$$

Instead of taking $U$ and $V$ to be uniform balls we can let $\rho: X \rightarrow R^{+}$ be a strictly positive lower-semi-continuous bounded function and let

$$
U=\{f \in C(X):|f(x)| \leqq \rho(x)\} .
$$

Thus, for example, if $\rho \equiv 1$ on $Y$ and $\rho \equiv \alpha(\alpha>1)$ on $X \backslash Y$ then the problem of finding, for $g \in A$ and uniform $\left\|\left.g\right|_{Y}\right\| \leqq 1$, an $f \in A$ such that $\left.f\right|_{Y}=\left.g\right|_{Y}$ and (uniform) $\|f\| \leqq \alpha$, is the interpolation problem for $\hat{Y}=K^{\circ} \cap U^{\circ}$, involving $A, U \cap A$ and $\left.U\right|_{Y}$.

In fact, following Roth [20] we may take $\rho: X \times T \rightarrow R^{+}(T$ the unit circle in $\mathrm{C}$ ) to be strictly positive, l.s.c. and bounded with

$$
U=\{f \in C(X): \operatorname{re} t f(x) \leqq \rho(x, t)\}
$$

We return to this in $\S 4$.

With the $C(X)$ example in mind we take, for $E$ a Banach space and $K$ a closed subspace,

$$
\hat{Y}=K^{\circ} \cap V^{\circ}: V \text { a 0-neighborhood in } E \text {. }
$$

Thus $\hat{Y}$ is an interpolation set for $E$ and $K^{\circ}$ is isomorphic to $A_{0}(\hat{Y})$.

If $\theta: E \rightarrow A_{0}(\hat{Y})$ is the restriction map then let $\hat{V}=\left\{f \in A_{0}(\hat{Y}): \operatorname{re}(f, y) \leqq 1\right.$ for all $\left.y \in \hat{Y}\right\} ;$ in other words, $\hat{V}$ is the polar of $\hat{Y}$ in the duality $A_{0}(\hat{Y}), A_{0}(\hat{Y})^{*}$.

We take $A$ to be a closed convex cone in $E$ and $B$ a closed bounded convex set containing 0 in $E$ such that $\pm B \subset A$.

Proposition 2.5.

(1) $\hat{V}=\left(\left.V\right|_{\hat{Y}}\right)^{-}$,

(2) $\left(\theta^{-1}\right)(\hat{V})=\overline{K+V}$.

Consider IP(*) involving $A, B$ and $\hat{V}$ :

(3) approximate solutions exists if and only if

$$
A \cap\left(K+V^{\prime}\right) \subset B^{\prime}+K,
$$

(4) exact solutions exist if and only if

$$
A \cap(K+V)-\subset B+K \text {. }
$$

Proof. First, $(\theta V)^{\circ}=\left(\theta^{*}\right)^{-1}\left(V^{\circ}\right)=\left(\theta^{*}\right)^{-1}\left(V^{\circ} \cap K^{\circ}\right)=\hat{Y}$ so (1) holds. Also, $\theta^{-1}(\hat{V})=\left(\theta^{*} \hat{Y}\right)^{\circ}=\left(K^{\circ} \cap V^{\circ}\right)^{\circ}=\overline{K+V}$.

Now (3), (4) follow from (1) and (2).

In the future we shall refer to $\operatorname{IP}(*)$ or $\operatorname{IP}(*)$ for $\hat{Y}$ with the under- 
standing that we are in the above set-up: $\hat{Y}=K^{\circ} \cap V^{\circ}$ and $\operatorname{IP}(*)$ is the interpolation problem for $\hat{Y}$ involving sets $A, B \subset E$ and $\hat{V}=(V \mid \hat{Y})^{-} \subset A_{0}(\hat{Y})$.

We next characterize the existence of approximate solutions in a fashion closely related to the closed-range conditions of Theorem 2.2.

Theorem 2.6. Let $A$ be a closed cone in $E$ and $B$ a closed bounded convex subset of $E$ containing 0 such that $\pm B \subset A$. Then, in the set-up of Proposition 2.5, the following are equivalent and imply that $A+K$ is closed:

(i) The interpolation problem $\mathrm{IP}(*)$ has approximate solutions,

(ii) $A \cap(\overline{K+V}) \subset \overline{B+K}$,

(iii) $(\overline{A+K}) \cap(\overline{K+V}) \subset \overline{B+K}$,

(iv) $(\overline{A+K}) \cap\left(K+V^{\prime}\right) \subset B^{\prime}+K$.

Conversely, if $A$ is a subspace and $A+K$ is closed then the above hold for some $B$ of the form $U \cap A ; U$ a 0-neighborhood.

Proof. The equivalence of (i) and (ii) (and (iii) with (iv)) is a consequence of the gauge lemma with

$$
D \longleftrightarrow A, A \longleftrightarrow K, B \longleftrightarrow B \text { and } C \longleftrightarrow V .
$$

For (ii) implies (iii) we note that if $x \in(\overline{A+K}) \cap(\overline{K+V})$ then

$$
x=a+k_{1}+v_{1}=k_{2}+v+v_{3} ; v_{1}, v_{3} \text { small }
$$

so that $a \in A \cap(K+V) \subset \overline{B+K})$. Since $v_{1}$ is arbitrarily small, $x \in \overline{B+K}$. The converse follows from the closed range theorem by taking the quotient map

$$
\theta: A \rightarrow A / K \text {. }
$$

We assume that the conditions of Theorem 2.6 hold:

$$
A \cap(K+V) \subset \overline{B+K}
$$

so that $A+K$ is closed and approximate solutions to the interpolation problem exist:

$$
(A+K) \cap(K+V)^{\prime} \subset B^{\prime}+K .
$$

We now formulate a further condition in $E$ that will yield the closure of $B+K$, thus providing exact solutions to the interpolation problem.

THEOREM 2.7. Let condition (1) above hold (and hence (1)') and assume further 


$$
\overline{B+K} \cap(1+r) B \subset \overline{B+r K \cap \bar{W}} \text { for any } r>0,
$$

where $W$ is a 0-neighborhood. Then $B+K$ is closed and hence

$$
(A+K) \cap(K+V) \subset B+K .
$$

In particular the interpolation problem $\mathrm{IP}(*)$ has exact solutions.

Proof. Let $D=A+K$. In view of $(1)^{\prime}, D$ is closed, $\pm B \subset D$, $K \subset D$ and $B+K$ is $D$-regular. Hence, from Corollary 1.3 we have $\overline{B+r \mathrm{~K} \cap W}$ is $D$-regular, so that (2) is equivalent to

$$
\overline{\mathrm{B}+K} \cap(1+r) B^{\prime} \subset B+r K \cap W .
$$

We now apply the gauge lemma:

$$
\begin{aligned}
& A \longleftrightarrow B, \\
& B \longleftrightarrow K \cap W, \\
& C \longleftrightarrow B, \\
& D \longleftrightarrow \overline{B+K} .
\end{aligned}
$$

Thus (3) implies condition (3) of the gauge lemma $(\alpha=1)$ and the other conditions are clearly met so that $\bar{d}=d$ on $D$. Hence, if $x \in \overline{B+K}$ then $x \in \overline{B+r K \cap \bar{W}}$ for some $r$. Therefore $x \in(1+\epsilon)$ $(B+r K \cap W)$ for any $\epsilon>0$ so that $\bar{d}(x) \leqq r$. The conclusion $d(x) \leqq r$ says $x \in B+r^{\prime} K \cap W$ (any $r^{\prime}>r$ ) so that $x \in B+K$. Hence $B+K$ is closed and, from (1)',

$$
(A+K) \cap(K+V) \subset \overline{B+K} \subset B+K,
$$

giving (2)' and the exactness of solutions to the interpolation problem.

An alternative approach to formulating conditions for abstract interpolation problems can be found in Roth [20]. In particular, a translation-invariance property of neighborhoods is used to assure exactness of solutions.

As an example o the geometric content of condition (2) suppose that $E=K \oplus L$ where $L$ is a closed subspace complementary to $K$. Take

$$
B=\left\{x=x_{1}+x_{2} ; x_{1} \in K_{1}, x_{2} \in L_{1}\right\} \text {. }
$$

Then if $x \in(B+K) \cap(1+r) B$ we have

$$
x=b+k=(1+r) b^{\prime}, b, b^{\prime} \in B, k \in K \text {. }
$$


Then $b_{1}, b_{1}{ }^{\prime} \in K_{1}, b_{2}, b_{2}{ }^{\prime} \in L_{1}$ and $b_{1}+k=(1+r) b_{1}{ }^{\prime}$. Thus

$$
x=b_{1}+k+b_{2}=b_{1}{ }^{\prime}+r b_{1}{ }^{\prime}+b_{2}=b_{1}{ }^{\prime}+b_{2}+r b_{1}{ }^{\prime} \in B+r K_{1} .
$$

Hence

$$
(B+K) \cap(1+r) B \subset B+r K_{1} .
$$

Thus, if we think of $E$ as $K \times L$ and $B$ as the unit ball of the $L^{\infty}$ norm of this product then condition (2) becomes quite natural. In the following sections we are concerned with polar conditions, in which case, pursuing this example, $E^{*}=K^{*} \times L^{*}$ has an $L^{1}$ norm on $B^{\circ}$. It will frequently happen that, although $K$ is not complemented in $E, K^{\circ}$ is complemented (in the norm topology) in $E^{*}$. This leads to the study of split sets and their generalizations, which we consider next.

3. Decomposable Sets and Split Sets. Let $S$ be a closed convex set containing 0 in the Banach space $E$. Let $K$ be a closed subspace and let $W$ be a neighborhood of 0 (not necessarily bounded) in $E$. The distance functional $d(K, W)$, measuring distance from $K$ via $W$, is just the Minkowski functional of $K+W$ and we will denote it by $d_{W}$, the $K$ being understood throughout. Let $p_{S}$ be the Minkowski functional of $S$.

Definition. The set $S$ is locally decomposable by $K$ if there is a positively homogeneous map $\pi: S \rightarrow S \cap K$ with the following properties:

(1) $\pi=1$ (the identity map) on $S \cap K$,

(2) $1-\pi: S \rightarrow S$,

(3) $p_{\mathrm{S}}=p_{1}+p_{2}$ where $p_{1}=p_{\mathrm{S}} \circ \pi$ and $p_{2}=p_{\mathrm{S}} \circ(1-\pi)$, and

(4) $p_{2} \leqq d_{W}$ on $S$ for some neighborhood $W$ of 0 .

Roughly speaking, $S$ is decomposable by $K$ if $S$ is the convex hull of $S \cap K$ and the portion of $S$ some positive distance away from $K$.

In fact, if $S$ is bounded then the following proposition shows this to be precisely the case.

Proposition 3.1. If $S$ is a closed and bounded convex set containing 0 in $E$ then $S$ is locally decomposable by the closed subspace $K$ if and only if each $x \in S$ is a convex combination of a point $y \in S \cap K$ and a point $z \in S$ with $d_{w}(z) \geqq 1$.

Proof. Let $T=\left\{z \in ; d_{W}(z) \geqq 1\right\}$ and let $S$ be decomposable by $K$. Let $p_{S}(x)=1$ and write

$$
x=x_{1}+x_{2} ; x_{1}=\pi x \in S \cap K \text { and } x_{2}=(1-\pi) x \in S .
$$

If $p_{1}(x)=p_{S}\left(x_{1}\right)=0$ then, since $S$ is bounded, $x=x_{2}$ and $p_{2}(x)=p_{S}(x)=1 \leqq d_{W}(x)$ so that $x \in T$. Similarly, if $p_{2}(x)=0$ then 
$x \in S \cap K$. If $0<p_{i}(x) ; i=1,2$, then

$$
\begin{aligned}
x & =p_{1}(x) y+p_{2}(x) z \text { where } \\
y & =x_{1} / p_{1}(x) \in S \cap K, z=x_{2} / p_{2}(x) \in S \text { and } \\
d_{W}(z) & =d_{W}\left(x_{2}\right) / p_{2}(x)=d_{W}(x) / p_{2}(x)\left(\text { since } x_{1} \in K\right) \geqq 1 .
\end{aligned}
$$

Conversely, if $x=\lambda y+(1-\lambda) z$ with $p_{S}(x)=1, y \in S \cap K$ and $z \in T$ then we define $\pi x=\lambda y$ and extend $\pi$ to be positively homogeneous. Properties (1)-(4) are easily verified.

We return to the interpolation problem $\mathrm{IP}(*)$ preceding Theorem 2.6 and formulate the conditions for approximate and exact solutions in terms of the dual space $E^{*}$. Let $d\left(A^{\circ}, \hat{Y}\right)$ be the distance from $A^{\circ}$ via $\hat{Y}$.

Theorem 3.2. If, in the interpolation problem $\operatorname{IP}(*)$, the set $B^{\circ}$ is locally decomposable by $\mathrm{K}^{\circ}$ under the map $\pi$, with

$$
d\left(A^{\circ}, \hat{Y}\right) \circ \pi \leqq 1 \text { on } B^{\circ}
$$

then $\operatorname{IP}(*)$ possesses exact solutions.

Proof. The polars of the conditions (1) and (2) of Theorem 2.7 are

$(1)^{\circ} B^{\circ} \cap K^{\circ} \subset A^{\circ}+\hat{Y}$,

$(2)^{\circ}(B+r K \cap W)^{\circ} \subset \operatorname{cl}-\operatorname{conv}\left(B^{\circ} \cap K^{\circ}, s B^{\circ}\right) ; s=1 /(1+r)$.

If $B^{\circ}$ is decomposable and $d\left(A^{\circ}, \hat{Y}\right) \circ \pi \leqq 1$ on $B^{\circ}$ then, since $\pi=1$ on $B^{\circ} \cap K^{\circ},(1)^{\circ}$ is satisfied.

Now $p_{B^{\circ}}=p_{1}+p_{2} ; p_{1}=p_{B^{\circ}} \circ \pi, p_{2}=p_{B^{\circ}} \circ(1-\pi)$ and $p_{2} \leqq d_{\hat{W}}$, where $\hat{W}$ is a neighborhood of 0 in $E^{*}$. Without loss of generality we can replace $\hat{W}$ by a smaller 0-neighborhood of the form $W^{\circ}$, where $W$ is a 0 -neighborhood in $E$. We show $(2)^{\circ}$ holds with this choice of $W$. The Minkowski functional, $h$, of $(B+r K \cap W)^{\circ}$ is the support functional of $B+r K \cap W$ and hence $h=\rho_{B}+r \rho_{K \cap W}$. But $\rho_{B}=p_{B^{\circ}}$ and $\rho_{K \cap W}=d_{W^{\circ}}$, so if $h$ is finite then so is $p_{B^{\circ}}$ and

$$
\begin{aligned}
h & =p_{B^{\circ}}+r d_{W^{\circ}}=p_{1}+p_{2}+r d_{W^{\circ}} \\
& \geqq p_{1}+p_{2}+r p_{2}=p_{1}+(1+r) p_{2} .
\end{aligned}
$$

Thus if $h(x) \leqq 1$ then $x=x_{1}+x_{2} ; x_{1}=\pi x, x_{2}=(1-\pi) x$ and

$$
p_{B^{\circ}}\left(x_{1}\right)+(1+r) p_{B^{\circ}}\left(x_{2}\right) \leqq 1 \text {. }
$$

Hence $x$ is in cl-conv $\left(B^{\circ} \cap K^{\circ},(1 /(1+r)) B^{\circ}\right)$.

In Theorem 3.2 the closure of $B+K$ in the conclusion required the closed range condition (1) in addition to the condition (2). We have seen that the local decomposability of $B^{\circ}$ by $K^{\circ}$ yields $(2)^{\circ}$ and hence (2). It is useful to have at hand a stronger decomposability property 
that will assure that $B+K$ is closed independent of condition (1). We first apply the gauge lemma in $E$ to derive a condition for the closure of $B+K$.

TheоReм 3.3. Let $E$ be a Banach space with unit ball $U, K$ a closed subspace and $B$ a closed convex subset containing 0 . If there exists a 0neighborhood $W$ such that

$$
(B+K)^{-} \cap(B+r U) \subset(B+r W \cap K)^{-} \text {for all } r>0
$$

then $B+K$ is closed in $E$.

Proof. Apply the gauge lemma with

$$
A \longleftrightarrow B, B \longleftrightarrow W \cap K, C \longleftrightarrow U, D \longleftrightarrow(B+K)^{-} .
$$

Thus we conclude that $d=\bar{d}$. But $x \in(B+K)^{-}$implies, by (*), that $\bar{d}(x)=r$ for some $r<\infty$. Hence $d(x)<r^{\prime}$ for any $r^{\prime}>r$, so that $x \in B+r^{\prime} W \cap K \subset B+K$.

Definition. A closed convex set $S$ containing 0 in $E$ is decomposable by $K$ if there exists a map $\pi: E \rightarrow K$ such that

(1) $\pi=1$ on $K$,

(2) $p_{S}=p_{1}+p_{2}$ where $p_{1}=p_{S} \circ \pi$ and $p_{2}=p_{S} \circ(1-\pi)$ and

(3) $p_{U} \circ(1-\pi) \leqq d_{W}$ on $S$ for some neighborhood $W$ of 0 .

Corollary 3.4. If $B^{\circ}$ is decomposable by $K^{\circ}$ in $E^{*}$ then $B+K$ is closed in $E$.

Proof. We show that, for some 0-neighborhood $W$,

$$
(B+r W \cap K)^{\circ} \subset \operatorname{cl}-\operatorname{conv}\left(B^{\circ} \cap K^{\circ},(B+r U)^{\circ}\right)
$$

holds for all $r>0$ : let $h$ be the Minkowski functional of $(B+r W \cap K)^{\circ}$ where $W^{\circ}$ satisfies $p_{U^{\circ}}{ }^{\circ}(1-\pi) \leqq d_{W^{\circ}}$ and $W^{\circ}$ is weak* compact. Then

$$
\begin{aligned}
h & =p_{B^{\circ}}+r d_{W^{\circ}} \geqq p_{1}+p_{2}+r p_{U^{\circ}} \circ(1-\pi) \\
& =p_{B^{\circ}} \circ \pi+\left(p_{B^{\circ}}+r p_{U^{\circ}} \circ(1-\pi) .\right.
\end{aligned}
$$

The condition $(*)^{\circ}$ now follows as in Theorem 3.2 and hence, by taking polars, (*) holds.

A particular instance of decomposability, which has received a great deal of attention in the literature, arises when the map $\pi$ is a bounded linear projection. In this case sets that are decomposable by $K$ under $\pi$ are called split sets. We note that in the definition of "decomposable", 
property (1) is automatic and property (3) follows as well, since the linearity of $\pi$ gives

$$
p_{U}^{\circ}(1-\pi) \leqq \beta d_{U}
$$

where $\beta$ is a bound for $1-\pi$.

We assume now that $E=K \oplus L$ with $\pi_{1}$ and $\pi_{2}$ the projections onto $K, L$ respectively with $1=\pi_{1}+\pi_{2}$. Then we say a closed convex set $S$ containing 0 is split, or split by $K$, if

$$
p_{S}=p_{1}+p_{2}
$$

where $p_{i}=p_{\mathrm{S}}{ }^{\circ} \pi_{i} ; i=1,2$.

Proposition 3.5. Let $A, B$ be closed convex subsets of $K, L$ respectively, each containing 0 . If $S=\operatorname{cl}-\operatorname{conv}(A, B)$ then

(1) $S=\operatorname{conv}(A, B)+P_{A}+P_{B}$ where

$$
P_{A}=\left\{a \in A: p_{A}(a)=0\right\}, P_{B}=\left\{b \in B: p_{B}(b)=0\right\},
$$

(2) $p_{\mathrm{S}}=p_{A} \circ \pi_{1}+p_{B} \circ \pi_{2}$.

Proof. Let $x_{n} \rightarrow x$ where $x_{n} \in \operatorname{conv}(A, B)$. Then

$$
\begin{gathered}
x_{n}=\lambda_{n} a_{n}+\mu_{n} b_{n}, \\
a_{n} \in A, b_{n} \in B \text { and } \lambda_{n}+\mu_{n} \leqq 1 .
\end{gathered}
$$

Thus $x_{1}=\pi_{1} x=\lim \lambda_{n} a_{n}$ and

$$
p_{A}\left(x_{1}\right) \leqq \lim \inf p_{A}\left(\lambda_{n} a_{n}\right) \leqq \lim \inf \lambda_{n}
$$

By taking a subsequence we can assume $\lambda_{n} \rightarrow \lambda, \mu_{n} \rightarrow \mu ; \lambda+\mu \leqq 1$ so that $x=x_{1}+x_{2}=\lambda\left(x_{1} / \lambda\right)+\mu\left(x_{2} / \mu\right)$, where we mean 1 for $0 / 0$. Hence (1) follows and therefore (2) as well.

Proposition 3.6. Let $E=K \oplus L$ and let $S$ be a closed convex set containing 0 . The following are equivalent:

(i) $\mathrm{S}$ is decomposable by $\mathrm{K}$ under $\pi_{1}$,

(ii) $\mathrm{S}$ is split,

(iii) $S=\operatorname{cl}-\operatorname{conv}(S \cap K, S \cap L)$,

(iv) $S=\operatorname{cl}-\operatorname{conv}\left(\pi_{1}(S), \pi_{2}(S)\right)$.

Proof. The equivalence of (i) and (ii) follows from the above comments, and the property $p_{\mathrm{S}}=p_{1}+p_{2}$ implies (iii). If (iii) holds then, since $\pi_{i}$ is continuous, $\pi_{1}(S) \subset S \cap K$ and $\pi_{2}(S) \subset S \cap L$. Hence equality holds and thus (iv) follows. Conversely, if (iv) holds $\pi_{i}(S) \subset S$ $(i=1,2)$ so that (iii) holds. Finally, (iii) implies (ii) and (i) by the preceding proposition. 
We are mainly interested in sets whose polars are split in the dual space. The linearity of the map $\pi$ allows us to conclude some stability properties of such sets under intersections.

THEOREM (ANDo [4]) 3.7. Let $K$ be a closed subspace of $E$ such that $E^{*}=K^{\circ} \oplus \hat{L}$ where $\pi_{1}$ and $\pi_{2}$ are the bounded projections onto $K^{\circ}, \hat{L}$ with $1=\pi_{1}+\pi_{2}$. Let $S, T$ be closed convex sets containing 0 in $E$ such that $S^{\circ}$ and $T^{\circ}$ are split by $K^{\circ}$ under $\pi_{1}$. Then $S+K$ and $T+K$ are closed in $E$. In addition,

(1) If either $S^{\circ}$ or $T^{\circ}$ is bounded then $(S \cap T)^{\circ}$ is split so that $S \cap T+K$ is closed.

(2) If $\left\|\pi_{2}\right\|=1$ then $(S \cap T+K)^{-} \subset S \cap(T+\epsilon U)+K$ for any $\epsilon>0$, where $U$ is the unit ball of $E$.

Proof. The preceding proposition shows $S+K$ and $T+K$ are closed in $E$. Now

$$
(\mathrm{S} \cap T)^{\circ}=\text { weak }^{*} \operatorname{cl}-\operatorname{conv}\left(\mathrm{S}^{\circ}, T^{\circ}\right) .
$$

If $S^{\circ}$ or $T^{\circ}$ is bounded then

$$
(\mathrm{S} \cap T)^{\circ}=\operatorname{cl}-\operatorname{conv}\left(S^{\circ}, T^{\circ}\right) \text { (norm closure). }
$$

But $\pi_{i}\left[\operatorname{cl-conv}\left(S^{\circ}, T^{\circ}\right)\right]=\operatorname{cl}-\operatorname{conv}\left(\pi_{i}\left(S^{\circ}\right), \pi_{i}\left(T^{\circ}\right)\right) ; i=1,2$. Hence, if $S^{\circ}$ or $T^{\circ}$ is bounded,

$$
\begin{aligned}
(\mathrm{S} \cap T)^{\circ} & =\operatorname{cl}-\operatorname{conv}\left(\pi_{1}\left(S^{\circ}\right), \pi_{1}\left(T^{\circ}\right), \pi_{2}\left(S^{\circ}\right), \pi_{2}\left(T^{\circ}\right)\right) \\
& =\operatorname{cl}-\operatorname{conv}\left(\pi_{1}\left((S \cap T)^{\circ}\right), \pi_{2}\left((S \cap T)^{\circ}\right)\right) .
\end{aligned}
$$

For (2) we let $R_{\epsilon}=S \cap(T+\epsilon U)$ with $h_{\epsilon}$ the Minkowski functional of $\left(R_{\epsilon}\right)^{\circ}, T_{\epsilon}=T+\epsilon U$ with $g_{\epsilon}$ the functional of $\left(T_{\epsilon}\right)^{\circ}$ and $\hat{R}=\operatorname{cl}-\operatorname{conv}\left(\mathrm{S}^{\circ}\right.$, $T^{\circ}$ ) (norm closure) with functional $h$. We show the polar of

$$
(S \cap T+K)^{-} \cap\left(R_{\epsilon}+r U\right) \subset\left(R_{\epsilon}+r U \cap K\right)^{-}
$$

holds, so that the conclusion follows from the gauge lemma with

$$
A \longleftrightarrow R_{\epsilon}, B \longleftrightarrow U \cap K, C \longleftrightarrow U, D \longleftrightarrow(S \cap T+K)^{-} .
$$

Thus we want

$$
\left(R_{\epsilon}+r U \cap K\right)^{\circ} \subset \operatorname{cl}-\operatorname{conv}\left[K^{\circ} \cap(S \cap T)^{\circ},\left(R_{\epsilon}+r U\right)^{\circ}\right] .
$$

First $g_{\epsilon}=p_{T^{\circ}}+\epsilon p_{U^{\circ}} \geqq p_{T^{\circ}} \circ \pi_{1}+\left(p_{T^{\circ}}+\epsilon p_{U^{\circ}}\right) \circ \pi_{2}$ (since $\left\|\pi_{2}\right\|=1$ ) so that $T_{\epsilon}^{\circ} \subset$ cl-conv $\left[T^{\circ} \cap K^{\circ},\left(T_{\epsilon}\right)^{\circ} \cap \hat{L}\right]$. Now 


$$
\begin{aligned}
\left(R_{\epsilon}\right)^{\circ} & =\operatorname{cl}-\operatorname{conv}\left[S^{\circ}, T_{\epsilon}^{\circ}\right](\text { norm closure) } \\
& =\operatorname{cl}-\operatorname{conv}\left[S^{\circ} \cap K^{\circ}, S^{\circ} \cap \hat{L}, T^{\circ} \cap K^{\circ}, T_{\epsilon}^{\circ} \cap \hat{L}\right] \\
& =\operatorname{cl}-\operatorname{conv}\left[\hat{R} \cap K^{\circ}, R_{\epsilon}^{\circ} \cap \hat{L}\right]
\end{aligned}
$$

so that $h_{\epsilon} \geqq h \circ \pi_{1}+h_{\epsilon} \circ \pi_{2}$. Hence

$$
\begin{aligned}
h_{\epsilon}+r d_{U^{\circ}} & \geqq h \circ \pi_{1}+h_{\epsilon}^{\circ} \pi_{2}+r p_{U^{\circ}} \circ \pi_{2} \\
& =h^{\circ} \pi_{1}+\left(h_{\epsilon}+r p_{U^{\circ}} \circ \pi_{2},\right.
\end{aligned}
$$

completing the proof.

4. The $C(X)$ Case. In this section we consider interpolation problems beginning with a compact Hausdorff space $X$ and a closed subset $Y$. We consider a closed subspace $A \subset C(X)$ such that $1 \in A$ and $A$ separates points of $X$. Let $K=\{f \in C(X): f \equiv 0$ on $Y\}$. Then $C(X)^{*} \cong \mathscr{M}(X)$, the space of regular Borel measures on $X$, and $K^{\circ}=\{\mu \in \mathscr{M}(x): \operatorname{supp} \mu \subset Y\} \cong C(Y)^{*}$. Clearly $K^{\circ}$ is the range of the projection $\pi, \pi \mu=\left.\mu\right|_{Y}$, and hence we have $C(X)^{*}=K^{\circ} \oplus \hat{L}$; $\hat{L}=\{\mu:|\mu|(Y)=0\}$. In many of the standard results the central idea involves the splittability of certain sets by the projection $\pi$.

To obtain results concerning "dominated" interpolation we deal with a fairly general gauge on $C(X)$. Following Roth [20] we let, for $T$ the unit circle in $\mathbf{C}$,

$$
\rho: X \times T \rightarrow R^{+}
$$

be a strictly positive, bounded, lower-semi-continuous function and

$$
U=\{f \in C(X): \operatorname{re} t f(x) \leqq \rho(x, t) ;(x, t) \in X \times T\} .
$$

Then the Minkowski functional of $U$ will be denoted by

$$
\|f\|_{\rho}=\sup \{\operatorname{ret} t f(x) / \rho(x, t):(x, t) \in X \times T\} .
$$

We shall also use $\|\cdot\|_{\rho}$ to denote the Minkowski functional of $U^{\circ}$ in $C(X)^{*}$.

Note that $\|\cdot\|_{\rho}$ is sub-additive and positive homogeneous but not necessarily absolutely homogeneous and hence is not precisely a norm for $C(X)$ in the usual sense. If $\rho \equiv 1$ then, of course, $\|\cdot\|_{\rho}$ is the uniform norm.

Let $\phi: X \times T \rightarrow C(X)^{*}$ be given by $\phi(x, t)(f)=\operatorname{ret} t f(x) / \rho(x, t)$.

Proposition (Roth) 4.1.

(1) $U$ is a 0-neighborhood in $C(X)$ and $U^{\circ}=w^{*} \operatorname{cl}-\operatorname{conv} \phi(X \times T)$.

(2) If $h$ is a bounded Borel function such that $\|h\|_{\rho} \leqq 1$ then $h \in U^{\circ \circ} \subset C(X)^{* *}$. 
(3) $U^{\circ}$ is split in $C(X)^{*}$ by $K^{\circ}$.

(4) $\left.U\right|_{Y}=\left\{g \in C(Y):\|g\|_{\rho} \leqq 1\right\}$.

Proof. (1) follows from the definition of $\rho$ and the separation theorem. For (2), let $\mu \in U^{\circ}$ be given and $\epsilon>0$. By Lusin's Theorem there is a $g \in C(X)$ and $F$ compact in $X$ such that

$$
\left.g\right|_{F}=\left.h\right|_{F},|\mu|(X \backslash F)<\epsilon / M ; M=2\|h\|_{\infty} \text { and }\|g\|_{\infty} \leqq\|h\|_{\infty} .
$$

For each $x \in X \backslash F$ choose, by Urysohn's Lemma, $\psi_{x} \in C(X)$ such that

$$
0 \leqq \psi_{x} \leqq 1, \psi_{x} \equiv 1 \text { on } F \text { and } \psi_{x}(x)=0 \text {. }
$$

Let $V_{x}=\left\{\left(x^{\prime}, t\right)\right.$ : re $\left.t \psi_{x}\left(x^{\prime}\right) g\left(x^{\prime}\right)<\rho\left(x^{\prime}, t\right)+\epsilon\right\}$. Then $V_{x}$ is a neighborhood of $F \cup\{x\}$ and hence $X$ is covered by $V_{x_{1}}, \cdots, V_{x_{n}}$. Let $\psi=\psi_{x_{1}} \wedge \cdots \wedge \psi_{x_{n}}$ and $f=\psi g$. Then either

$$
\text { re } t f(x) \leqq 0<\rho(x, t)
$$

or

$$
0 \leqq \operatorname{ret} f(x)=\psi(x) \text { re } \operatorname{tg}(x) \leqq \psi_{x_{i}}(x) \text { re } \operatorname{tg}(x)<\rho(x, t)+\epsilon .
$$

Hence $f \in(1+\epsilon) U$ and

$$
\operatorname{re} \mu(h)=\operatorname{re} \mu(f)+\operatorname{re} \int_{X \backslash F}(h-f) d \mu \leqq(1+\epsilon)+\epsilon .
$$

Thus $h \in U^{\circ \circ}$. For (3) let $\pi_{1} \mu=\pi \mu=\left.\mu\right|_{Y}$ and $\pi_{2} \mu=\left.\mu\right|_{X \backslash Y}$. Choose $f_{i} \in U(i=1,2)$ such that $\operatorname{re}\left(f_{i}, \pi_{i} \mu\right) \geqq\left\|\pi_{i} \mu\right\|_{\rho}-\epsilon$. Let $h=$ $f_{1} \chi_{Y}+f_{2} \chi_{(X \backslash Y)}$. Then by (2),

$$
\|\mu\|_{\rho} \geqq \operatorname{re}(h, \mu) \geqq\left\|\pi_{1} \mu\right\|_{\pi}+\left\|\pi_{2} \mu\right\|_{\rho}-2 \epsilon .
$$

For (4), let $V=\left\{g \in C(Y):\|g\|_{\rho} \leqq 1\right\}$. Clearly $\left.U\right|_{Y} \subset V$ and hence (for $\theta$ the restriction map) $V^{\circ} \subset(\theta U)^{\circ}=\left(\theta^{*}\right)^{-1}\left(U^{\circ} \cap K^{\circ}\right)$. Conversely, if $\mu \in U^{\circ} \cap K^{\circ}$ and $g \in V$, then extend $g$ to $h \equiv 0$ on $X \backslash Y$. By (2),

$$
\text { re } \int_{Y} g d \mu=\operatorname{re} \int_{X} h d \mu \leqq 1
$$

so that $\mu \in V^{\circ}$. Hence $V=\overline{\theta U}$. But Theorem 3.7, together with (3), shows $U+K$ is closed and hence $V=\theta U$.

Now we focus on the interpolation problem $\operatorname{IP}(* *)$ involving $A$ and $A \cap U:$

$\operatorname{IP}(* *)$

$$
\text { given } g \in A \text { with }\left\|\left.g\right|_{Y}\right\|_{\rho} \leqq 1 \text { find } f \in A
$$

such that $\left.f\right|_{Y}=\left.g\right|_{y}$ and $\|f\|_{\rho} \leqq 1$. 
Let $q: C(X)^{*} \rightarrow C(X)^{*} / A^{\circ} \cong A^{*}$ be the quotient map. Then take $\hat{X}=q\left(U^{\circ}\right), \hat{Y}=q\left(U^{\circ} \cap K^{\circ}\right)$ and $\hat{K}=q\left(K^{\circ}\right)$. Thus

$$
\hat{X}=\operatorname{cl} \text {-conv } q^{\circ} \phi(X \times T) \quad\left(q^{\circ} \phi\right. \text { is "gauge" evaluation in }
$$

$\left.A^{*}\right)$,

$$
\begin{aligned}
& \hat{Y}=\operatorname{cl-conv} q^{\circ} \phi(Y \times T) \text {, and } \\
& \hat{K}=\bigcup_{n=1}^{\infty} n \hat{Y} .
\end{aligned}
$$

Finally, let

$$
\hat{V}=\left\{g \in A_{0}(\hat{Y}): \operatorname{re}(g, y) \leqq 1 \text { for all } y \in \hat{Y}\right\}
$$

(the polar in $A_{0}(\hat{Y})$ of $\hat{Y}$ ).

Clearly $\operatorname{IP}(* *)$ is just an equivalent reformulation of $\operatorname{IP}(*)$ involving $A$, $A \cap U$ and $V=\left.U\right|_{Y} \subset A_{0}\left(U^{\circ} \cap K^{\circ}\right)$. We define $Y$ to be an interpolation set for $A \cap U$ if $\operatorname{IP}(* *)$ has approximate solutions, or equivalently, if $\operatorname{IP}(*)$ involving $A, A \cap U, V$ has approximate solutions.

We say $Y$ is an exact interpolation set for $A \cap U$ if $\operatorname{IP}(*)$ (or $(* *))$ has exact solutions.

THEOREM 4.2. THe following are equivalent:

(1) $Y$ is an interpolation set for $A \cap U$,

(2) $\hat{Y}=\hat{X} \cap \hat{K} ; \hat{Y}$ is an interpolation set in $A^{*}$ for $A$ and $\operatorname{IP}(*)$ involving $A, A \cap U$ and $V$ has approximate solutions,

(3) $\left\|\mu+A^{\circ} \cap K^{\circ}\right\|_{\rho}=\left\|\mu+A^{\circ}\right\|_{\rho}$ for all $\mu \in K^{\circ}$,

(3) $\left\|\pi_{1} m+A^{\circ} \cap K^{\circ}\right\|_{\rho} \leqq\left\|-\pi_{2} m\right\|_{\rho}$ for all $m \in A^{\circ}$.

These conditions imply the following equivalent conditions:

(4) $A+K$ is closed in $E$,

(5) $A^{\circ}+K^{\circ}$ is weak ${ }^{*}$ closed in $E^{*}$,

(6) $\hat{K}$ is weak* closed in $A^{*}$.

Conversely, if (4), (5), or (6) hold then (1), (2) and (3) hold for U defined by the function $\rho$,

$$
\rho(x, t)=\left\{\begin{array}{l}
1 \text { for } x \in Y \\
r \text { for } x \in X \backslash Y \text { for some } 1 \leqq r<\infty .
\end{array}\right.
$$

Proof. From Theorem 2.6, (1) is equivalent to

$$
\overline{(A+K)} \cap \overline{(U+K)=A} \cap \overline{U+K,}
$$

which, by polars, is equivalent to 


$$
K^{\circ} \cap\left(A^{\circ}+U^{\circ}\right)=K^{\circ} \cap A^{\circ}+K^{\circ} \cap U^{\circ},
$$

which, by applying $q$, is equivalent to

$$
\hat{Y}=\hat{X} \cap \hat{K} \text {. }
$$

This in turn, by polars in $A, A^{*}$, is equivalent (Theorem 2.6 again) to the second statement in (2). Statement (3) is just a reformulation of $(1)^{\prime}$ using $\|\cdot\|_{\rho}$ to denote $p_{U^{\circ}}$. The equivalence of (3) and (3)' is a consequence of $U^{\circ}$ being split by $K^{\circ}$.

Theorem 2.6 again shows that (4) follows from (1) and that conversely (4) implies (1) for the appropriate choice of $\rho$. Thus (4) is equivalent to

$$
\hat{X} \cap \hat{K} \subset r \hat{Y} \text { for some } r \geqq 1 .
$$

But this is equivalent to (6) (by Theorem 2.2) which implies (5). Finally (5) implies $\left(A^{\circ}+K^{\circ}\right) \cap U^{\circ}$ is weak* compact and hence $\hat{K} \cap \hat{X}$ is weak* compact so that (6) holds by the Krein-Smulyan Theorem.

We say $Y$ is a full interpolation set for $A \cap U$ if $Y$ is an interpolation set for $A \cap U$ and $\left.A\right|_{Y}=C(Y)$.

CoRollary 4.3. The following are equivalent:

(1) $Y$ is a full interpolation set for $A \cap U$,

(2) $A+K=C(X)$

(3) $A^{\circ} \cap K^{\circ}=\{0\}$ and $A^{\circ}+K^{\circ}$ is weak closed in $C(X)^{*}$,

(4) $\left\|\pi_{1} m\right\|_{\rho} \leqq\left\|-\pi_{2} m\right\|_{\rho}$ for all $m \in A^{\circ}$.

Proof. (1) holds if and only if $A_{0}(\hat{Y}) \cong\left(C(Y),\left.\quad U\right|_{Y}\right)$. But $A_{0}(\hat{Y})^{*}=C(Y)^{*} /\left(A^{\circ} \cap K^{\circ}\right)$ so the result follows.

If the unit ball $\hat{X}$ is decomposable by $\hat{Y}$ then Theorem 3.2 yields exact solutions to the interpolation problem. We note below some measure-theoretic conditions that insure the decomposability of $\hat{X}$ by $\hat{Y}$.

Decomposability also enters into formulation of sufficient conditions for $Y$ to be a generalized peak set of $X$. (We say $Y$ is a generalized peak set of $X$ with respect to $A$ if for each neighborhood $V \supset Y$ there is an $f_{V} \in A$ such that $\left\|f_{V}\right\|_{\infty}=1=f(Y)$ and $|f|<1$ on $X \backslash V$.) As in [6] the cone $P_{\beta}$ is defined by

$$
P_{\beta}=\left\{f \in A: \operatorname{re} f \geqq \beta_{0}|f|\right\} ; 0<\beta_{0}<1
$$

and the dual cone is denoted

$$
P_{\alpha}{ }^{*}=\left\{x \in A^{*}: \operatorname{re}(f, x) \geqq 0 \text { for all } f \in P_{\beta}\right\} .
$$

Suppose that in the definition of "decomposable", the neighborhood 
$W$ in $A^{*}$ is of the form

$$
W=\left\{x \in A^{*}: \operatorname{re}(x, h) \leqq 1\right\} \text { for some } h \in(\hat{K})^{\circ} \text { in } A^{* *} .
$$

Then condition (3) of "decomposable" becomes

$$
p_{\hat{X}} \circ(1-\pi) \leqq \operatorname{re} h \text { on } P_{\alpha}^{*} \text {. }
$$

In this event we say $P_{\alpha}{ }^{*}$ is positively decomposable by $\hat{K}$. This is the concept of decomposability of cones in [6] where it is shown that if $P_{\alpha}{ }^{*}$ is (positively) decomposable by $\hat{K}$ then $Y$ is a generalized peak set.

To convert split set conditions in $C(X)^{*}$ to decomposability in $A^{*}$ we apply the quotient map

$$
q: C(X)^{*} \rightarrow C(X)^{*} / A^{\circ} \cong A^{*}
$$

Assume now we have a Banach space $E$ with closed subspaces $A, K$ such that

(a) $E^{*}=K^{\circ} \oplus \hat{L}$ with corresponding projections $\pi_{1}$ and $\pi_{2}$,

(b) $U$ is a 0 -neighborhood in $E$ with $U^{\circ}$ split in $E^{*}$. Denote the Minkowski functionals of $U, U^{\circ}$ by $\|\cdot\|_{\rho}$ and take $U$ such that $\|f\|_{\rho}=\|-f\|_{\rho}$ to avoid technical complications in using the triangle inequality.

(c) $q: E^{*} \rightarrow E^{*} / A^{\circ} \cong A^{*}$ is the quotient map.

Let

$$
\hat{X}=q\left(U^{\circ}\right), \hat{Y}=q\left(U^{\circ} \cap K^{\circ}\right) \text { and } \hat{K}=q\left(K^{\circ}\right)=\langle\hat{Y}\rangle \text {. }
$$

Lemma 4.4. If $\mu \in E^{*}$ satisfies $\|\mu\|_{\rho}=\left\|\mu+A^{\circ}\right\|_{\rho}$ then

$$
\left\|\pi_{i} \mu\right\|_{\rho}=\left\|\pi_{i} \mu+A^{\circ}\right\|_{\rho} ; i=1,2 .
$$

Proof. If $m \in A^{\circ}$ and $\|\mu\|_{\rho}=\left\|\mu+A^{\circ}\right\|_{\rho}$ then (for $i=1$ )

$$
\begin{aligned}
\left\|\pi_{1} \mu+m\right\|_{\rho} & =\left\|(\mu+m)-\pi_{2} \mu\right\|_{\rho} \geqq\|\mu+m\|_{\rho}-\left\|\pi_{2} \mu\right\|_{\rho} \\
& \geqq\|\mu\|_{\rho}-\left\|\pi_{2} \mu\right\|_{\rho}=\left\|\pi_{1} \mu\right\|_{\rho} .
\end{aligned}
$$

Thus $\left\|\pi_{1} \mu+A^{\circ}\right\|_{\rho}=\left\|\pi_{1} \mu\right\|_{\rho}$ and of course the case $i=2$ is the same.

TheOREM 4.5. Let $B$ be a closed convex set containing 0 in $E$ such that

(a) $B^{\circ}$ is split in $E^{*}$,

(b) $B^{\circ}+A^{\circ}$ is weak* closed in $E^{*}$,

(c) there is an $\alpha \geqq 1$ such that for all $\mu \in B^{\circ}$ and $\|\mu\|_{\rho}=\left\|\mu+A^{\circ}\right\|_{\rho}$, then $\left\|\pi_{2} \mu\right\|_{\rho} \leqq \alpha\left\|\pi_{2} \mu+\pi_{2} A^{\circ}\right\|_{\rho}$. Then $\hat{B}=q\left(B^{\circ}\right)$ is a weak* closed set in $A^{*}$ decomposable by $\hat{K}$.

Proof. We have $\hat{B}$ is weak* closed by $(b)$. If $p(\hat{B})$ is the Minkowski 
functional of $\hat{B}$ in $A^{*}$ then $p(\hat{B}) \circ q=p\left(B^{\circ}+A^{\circ}\right)$, the Minkowski functional of $A^{\circ}+B^{\circ}$ in $\mathrm{E}^{*}$. Given $x \in \hat{B}$ choose $\mu \in B^{\circ}$ such that $q \mu=x$ and (since $B^{\circ}+A^{\circ}$ is closed)

$$
p(\hat{B}) x=p\left(B^{\circ}+A^{\circ}\right) \mu=p\left(B^{\circ}\right) \mu .
$$

Let $\mu=\pi_{1} \mu+\pi_{2} \mu$ so that $p\left(B^{\circ}\right) \mu=p\left(B^{\circ}\right) \pi_{1} \mu+p\left(B^{\circ}\right) \pi_{2} \mu$ and define $\hat{\pi}_{1}=$ $q\left(\pi_{1} \mu\right), \quad \hat{\pi}_{2}=1-\hat{\pi}_{1}$. Clearly $\hat{\pi}_{1}$ can be chosen to be positive homogeneous and we have

$$
\begin{aligned}
p(\hat{B}) \hat{\pi}_{1} x+p(\hat{B}) \hat{\pi}_{2} x & =p\left(B^{\circ}+A^{\circ}\right) \pi_{1} \mu+p\left(B^{\circ}+A^{\circ}\right) \pi_{2} \mu \\
& \leqq p\left(B^{\circ}\right) \pi_{1} \mu+p\left(B^{\circ}\right) \pi_{2} \mu \\
& =p\left(B^{\circ}\right) \mu=p\left(B^{\circ}+A^{\circ}\right) \mu=p(\hat{B}) x
\end{aligned}
$$

Thus it remains to show $p(\hat{X}) \hat{\pi}_{2} x \leqq d_{W}(x)$ where we take $W=(1 / \alpha) \hat{X}$. Then

$$
p(\hat{X}) \hat{\pi}_{2} x=p\left(U^{\circ}+A^{\circ}\right) \pi_{2} \mu=\left\|\pi_{2} \mu+A^{\circ}\right\|_{\rho}=\left\|\pi_{2} \mu\right\|_{\rho}
$$

and

$$
\begin{aligned}
d_{W}(x) & =p\left((1 / \alpha) U^{\circ}+K^{\circ}+A^{\circ}\right) \mu=p\left((1 / \alpha) \pi_{2} U^{\circ}+\pi_{2} A^{\circ}\right) \pi_{2} \mu \\
& =\alpha\left\|\pi_{2} \mu+\pi_{2} A^{\circ}\right\|_{\rho} .
\end{aligned}
$$

For the following refinement we take $N$ to be a closed convex cone in $A$ and consider measure theoretic conditions for exact solutions to the interpolation problem $\operatorname{IP}(* *)$ involving $A$ and $N \cap U$.

Theorem 4.6. Let $\mu \in N^{\circ}$ imply that $\pi_{1} \mu \in A^{\circ}$. Then

(1) $N^{\circ}$ is split in $C(X)^{*}$,

(2) $N+\hat{X}$ is split by $\hat{Y}$ under the projections $\hat{\pi}_{i}$ defined by $\hat{\pi}_{i}^{\circ} q=q^{\circ} \pi_{i}(i=1,2)$,

(3) for each $\alpha_{0}, 0<\alpha_{0}<1$, the cone $\hat{P}_{\alpha}{ }^{*}$ is positively split by $\hat{K}$,

(4) the set $Y$ is a generalized peak set for which $\operatorname{IP}(* *)$ involving $A$ and $N \cap U$ has exact solutions for each choice of $U$ in $C(X)$ such that $U^{\circ}$ is split in $C(X)^{*}$.

Proof. Since $N \subset A, A^{\circ} \subset N^{\circ}$. Thus the condition implies that $\pi_{1} \mu$ and $\pi_{2} \mu=\mu-\pi_{1} \mu$ belong to $N^{\circ}$ so that $N^{\circ}=\pi_{1} N^{\circ}+\pi_{2} N^{\circ}$ and hence is split.

Furthermore, $\mu \in A^{\circ}$ implies $\pi_{i} \mu \in A^{\circ}$ so that $q \circ \pi_{i}=0$ on $A^{\circ}$. Hence $\hat{\pi}_{i}(i=1,2)$ is well-defined with $\hat{\pi}_{1}$ a projection onto $\hat{K}$. Thus $U^{\circ}+N^{\circ}$ split in $C(X)^{*}$ implies $\hat{X}+N$ split in $A^{*}$ and $(\hat{X}+\hat{N}) \cap \hat{K}=\hat{Y}$ follows from the corresponding relation in $C(X)^{*}$. The exactness of solutions to the interpolation problem $\left.A\right|_{Y}, N \cap U$ now 
follows.

Since the cone $P_{\alpha}{ }^{*}$ is positively split by $K^{\circ}$ (see [6]) the property is preserved under $q$ so that $\hat{P}_{\alpha}{ }^{*}$ is positively split by $\hat{K}$. Thus [6] shows $Y$ is a generalized peak set.

Corollary 4.7. Let $\mu \in N^{\circ}$ imply $\pi_{1} \mu=0$. Then $Y$ is a generalized peak set and an exact full interpolation set for $N \cap U$.

The following weaker properties allow us to conclude the decomposability of the appropriate sets in $A^{*}$ and in consequence, conclusions similar to Theorem 4.6.

(a) $\left\|\pi_{1} m+A^{\circ} \cap K^{\circ}\right\|_{\rho} \leqq\left\|\pi_{2} m\right\|_{\rho}$ for all $m \in A^{\circ}$,

(b) $\left\|\pi_{1} m+A^{\circ}\right\|_{\rho} \leqq s\left\|\pi_{2} m\right\|_{\rho}(0 \leqq s<1)$ for all $m \in A^{\circ}$,

(c) $\left\|\pi_{1} m+A^{\circ} \cap K^{\circ}\right\|_{\rho} \leqq s\left\|\pi_{2} m\right\|_{\rho}(0 \leqq s<1)$ for all $m \in A^{\circ}$,

(d) $|m(Y)| \leqq s\left\|\pi_{2} m\right\|_{\rho}(0 \leqq s<1)$ for all $m \in A^{\circ}$.

We note that (c) implies both (a) and (b). Also (b) implies (d) since $m(Y)=\pi_{1} m(1)=\left(\pi_{1} m+n\right)(1)$ for all $n \in A^{\circ}$.

THEOREM 4.8. If properties (a) and (b) hold then $\hat{X}$ is decomposable by $\hat{Y}$ in $A^{*}$, and $Y$ is an exact interpolation set for $A \cap U$. If $P \leqq 1$ on $X \times T$ and $\equiv 1$ on $Y \times T$ then the A-convex hull of $Y$ is a generalized peak set.

Proof. If (a) holds then $\hat{X} \cap \hat{K}=\hat{Y}$ and we use Theorem 4.5 with $B=U$ to show $\hat{X}$ is decomposable at $\hat{Y}$. Consider $\mu \in C(X)^{*}$ with

$$
\|\mu\|_{\rho}=\left\|\mu+A^{\circ}\right\|_{\rho} \text {. Then }\left\|\pi_{2} \mu\right\|_{\rho}=\left\|\pi_{2} \mu+A^{\circ}\right\|_{\rho} .
$$

Given any $m \in A^{\circ}$, choose $n \in A^{\circ}$ such that $\left\|\pi_{1} m+n\right\|_{\rho} \leqq s\left\|\pi_{2} m\right\|_{\rho}$. Then

$$
\begin{aligned}
s\left\|\pi_{2} \mu+\pi_{2} m\right\|_{\rho} \geqq & s\left\|\pi_{2} m\right\|_{\rho}-s\left\|\pi_{2} \mu\right\|_{\rho} \geqq \| \pi_{1} m \\
& +n\left\|_{\rho}-s\right\| \pi_{2} \mu \|_{\rho} \\
= & \left\|\left(m-\pi_{2} m\right)+n\right\|_{\rho}-s\left\|\pi_{2} \mu\right\|_{\rho} \\
= & \left\|\left(\pi_{2} \mu+m+n\right)-\left(\pi_{2} m+\pi_{2} \mu\right)\right\|_{\rho}-s\left\|\pi_{2} \mu\right\|_{\rho} \\
\geqq & \left\|\pi_{2} \mu+m+n\right\|_{\rho}-\left\|\pi_{2} m+\pi_{2} \mu\right\|_{\rho}-s\left\|\pi_{2} \mu\right\|_{\rho} \\
\geqq & (1-s)\left\|\pi_{2} \mu\right\|_{\rho}-\left\|\pi_{2} m+\pi_{2} \mu\right\|_{\rho} .
\end{aligned}
$$

Therefore,

$$
\left\|\pi_{2} \mu\right\|_{\rho} \leqq[(1+s) /(1-s)]\left\|\pi_{2} \mu+\pi_{2} m\right\|_{\rho} .
$$

Thus Theorem 4.5 yields the decomposability of $\hat{X}$ by $\hat{Y}$. Property (d) is shown in [6] to establish the positive decomposability of $\hat{P}_{\alpha}^{*}$ at $\hat{K}$ for 
some $\alpha_{0}$ and the remaining conclusions follow.

In the event $A$ is an algebra (a subspace closed under pointwise multiplication of functions) in $C(X)$ things coalesce very nicely.

TheOREM 4.9. Let $A$ be an algebra in $C(X)$. Then the following are equivalent:

(a) $Y$ is a generalized peak set,

(b) $\pi_{1} \mu \in A^{\circ}$ for all $\mu \in A^{\circ}$,

(c) $\hat{X}$ is split by $\hat{Y}$ and $\hat{P}_{\alpha}{ }^{*}$ is positively split by $\hat{K}$ for each $\alpha_{0}$, $0<\alpha_{0}<1$,

(d) $Y$ is a generalized peak set and an exact interpolation set for each $A \cap U$ such that $U^{\circ}$ is split in $C(X)^{*}$,

(e) $\hat{X}$ is decomposable by $\hat{Y}$ and $\hat{P}_{\alpha}^{*}$ is positively decomposable by $\hat{K}$ for some $\alpha_{0}, 0<\alpha_{0}<1$.

Proof. Let (a) hold and let $\mu \in A^{\circ}$. We show $\int_{X} g d(\pi, \mu)$ $=\int_{Y} g d \mu=0$ for any $g \in A$. Given $\epsilon>0$, take any neighborhood $V$ of $Y$ such that $|\mu|(U \backslash Y)<\epsilon$ and $f \in A$ such that

$$
\text { (uniform }\|f\|=1=f(Y) \text {. }
$$

By replacing $f$ with $f^{n}$ for $n$ sufficiently large, we can assume $|f|<\epsilon$ on $X \backslash V$. Then

$$
\begin{aligned}
\int_{Y} g d \mu & =\int_{Y} g f^{n} d \mu=\int_{X} g f^{n} d \mu-\int_{X \backslash Y} g f^{n} d \mu \\
& =-\int_{X \backslash Y} g f^{n} d \mu .
\end{aligned}
$$

Thus

$$
\begin{aligned}
\left|\int_{Y} g d \mu\right| & \leqq \int_{X \backslash U}\left|g f^{n}\right| d|\mu|+\int_{U \backslash Y}\left|g f^{n}\right| d|\mu| \\
& \leqq \epsilon\|g\|\|\mu\|+\|g\||\mu|(U \backslash Y) .
\end{aligned}
$$

Then (b) implies (c) and (c) implies (d) follow from Theorem 4.6 and clearly imply (e). But (e) implies (a) by Theorem 4.8.

Corollary 4.10. Let $A$ be an algebra in $C(X)$. The following are equivalent:

(a) $\pi, \mu=0$ for all $\mu \in A^{\circ}$,

(b) $Y$ is a generalized peak set and a full exact interpolation set for each $A \cap U$ such that $U^{\circ}$ is split in $C(X)^{*}$.

The development of the results in this section essentially reverses the history of the subject. The first results, due to Rudin [21], Carleson [12] 
consist of Corollary 4.10 in the case $A$ is the disk algebra as a subspace of $C(T), T$ the circle in $C$. There, condition (a) is shown to hold if and only if $Y$ is a subset of $T$ with Lebesgue measure zero, a consequence of the $F$. and $M$. Riesz Theorem $[18,22]$.

The fact that approximate solutions can in some instances be made exact is shown here to be a consequence of decomposability in $A^{*}$ and thus relies on the iteration process in the gauge lemma. This technique appears in Bishop [9], Glicksberg [16], and Gamelin [15]. The measure theoretic characterization (b) if and only if (a) for generalized peak sets in Theorem 4.9 was proved in [16]. The equivalence of (a) and (c) in Theorem 4.9 was shown in a somewhat different form by Ellis [13] and, in the case of peak points, Asimow [5].

The use of property (c) (preceding Theorem 4.8) is due to Gamelin [15] where the number $s$ is called the extension constant.

The same property (c) appears in a general study of split sets in Ando [4], where results are also related to simultaneous linear interpolation. Results related to Theorem 4.6 have been formualted quite extensively. The measure condition there can be restricted to boundary measures for $A$ and Roth [20] shows that results of Alfsen-Hirsberg [2], Björk [10] and, in the real case, Andersen [3] follow from the observation that the quotient map $q$ preserves the projections in $C(X)^{*}$. A converse of Theorem 4.6 is also valid (see [20] for example).

Finally, the general criteria (3) and $(3)^{\prime}$ of Theorem 4.2 for interpolation sets can be found in Glicksberg [16].

\section{REFERENCES}

1. E. M. Alfsen, Compact convex sets and boundary integrals, Springer-Verlag Ergebnisse der Mathematik 57 (1971).

2. E. M. Alfsen and B. Hirsberg, On dominated extensions in linear subspaces in $C_{C}(X)$, Pacific J. Math. 36 (1971), 567-584.

3. T. B. Andersen, On dominated extension of continuous affine functions on split faces, Math. Scand. 29 (1971), 298-306.

4. T. Ando, Closed range theorems for convex sets and linear liftings, Pacific J. Math. 44 (1973), 393-409.

5. L. Asimow, Decomposable compact convex sets and peak sets for function spaces, PAMS 25 (1970), 75-79.

6. _ـ Exposed faces of dual cones and peak-set criteria for function spaces, J. Func. Anal. 12 (4) (1973), 456-474.

7. Complementary cones in dual Banach spaces, Illinois J. Math. 18 (4) (1974), 657-668.

8. Best approximation by gauges on a Banach space, J. Math. Anal. and Appl. 62 (1978), 571-580.

9. E. Bishop, A general Rudin-Carleson theorem, PAMS 13 (1962), 140-143.

10. J. E. Björk, Interpolation on closed subspaces of $C(X)$ (to appear). 
11. E. Briem, Interpolation on closed subspaces of $C(X)$, Journal of Functional Analysis 12 (1973), 1-12.

12. L. Carleson, Representations of continuous functions, Math. Z. 66 (1957), 447-451.

13. A. J. Ellis, Split faces and function algebras, Math. Ann. 195 (1972), 159-166.

14. T. W. Gamelin, Uniform Algebras, Prentice Hall, Englewood Cliffs, N.J., 1969.

15. —_ Restrictions of subspaces of $C(X)$, TAMS 112 (1964), 278-286.

16. I. Glicksberg, Measures orthogonal to algebras and sets of anti-symmetry, TAMS 105 (1962), 415-435.

17. O. Hustad, A norm preserving complex Choquet theorem, Math. Scand. 29 (1971), 272-278.

18. G. M. Leibowitz, Lectures on Complex Function Algebras, Scott-Foreman, Glenview, Ill., 1970.

19. F. Perdrizet, Espaces de Banach ordonnés et idéaux, J. Math. Pures Appl. 49 (1970), 61-98.

20. W. Roth, A general Rudin-Carleson theorem in Banach spaces, Pac. J. Math. 73 (1977), 197-213.

21. W. Rudin, Boundary values of continuous analytic functions, PAMS 7 (1956), 808-811.

22. E. L. Stout, The Theory of Uniform Algebras, Bogden and Quigley, Tarrytown-onHudson, N.Y., 1971.

Department of Mathematics, University of Wyoming, Laramie, WY 82071 